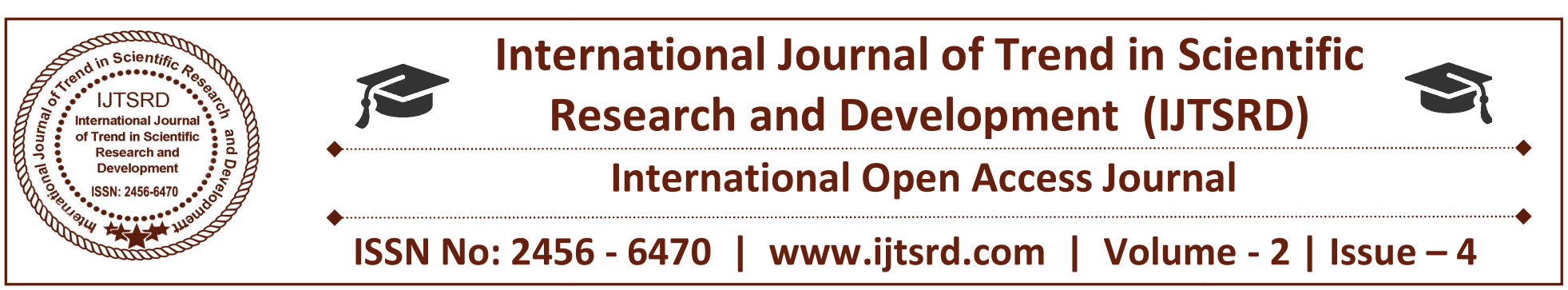

\title{
Comparative Analysis of AODV Base and RSSI Base Wireless Sensor Node Localization Techniques
}

\author{
Miss. Prajakta B. Patil ${ }^{1}$, Dr. A. N. Jadhav ${ }^{2}$ \\ ${ }^{1}$ PG Student, ${ }^{2}$ Principal and Professor \\ Department of Electronics and Telecommunication Engineering, \\ D. Y. Patilcollege of Engineering and Technology, \\ Shivaji University, Kolhapur, Maharashtra, India
}

\section{ABSTRACT}

Measurement-based node localization algorithms exploit the measurements of the received signals' characteristics such as RSSI (Received signal strength). Using the RSS measurement, the distance between any sensors' pair could be obtained by converting the power loss due to propagation from a sensor to another based on some propagation laws. Unfortunately, due to the probable presence of noise and interference, the distance's estimate would befar from being accurate, thereby leading to unreliable localization accuracy. The proposed approach can provide the deployment uniformly of known location nodes among the sensor fields and the lower computational complexity of path planning compared with method which utilizes only mobile nodes on the basis of a random movement. The results are compared with the AODV based basic localization technique with new RSSI based AODV node localization technique. Further analysis of the power consumption, PDR and throughput is done and shows satisfactory results.

Keywords: wireless sensor networks $\left(W S N_{S}\right)$, localization, node connectivity, RSS (received signal strength), node distance.

\section{INTRODUCTION}

Wireless Sensor Network (WSN) is currently a popular information acquisition and processing technology and one of the research hotspots. WSN technology integrates sensor, wireless network communication and embedded computing technologies and transmits data and information through WSN nodes[6].Generally, the localization algorithms have

been Proposed, which can be mainly classified into two categories: range-based and range-free. Several range-based techniques estimate an unknown node distance by three or more beacon nodes. Based on the range information, the location of a node is determined. Some of the range-based localization algorithms include received signal strength indicator (RSSI), angle of arrival (AOA), time of arrival (TOA), and time difference of arrival (TDOA) [4]. . The proposed approach can provide the deployment uniformly of known location nodes among the sensor fields and the lower computational complexity of path planning compared with method which utilizes only mobile nodes on the basis of a random movement. The results are compared with the AODV based basic localization technique with new RSSI based AODV node localization technique. Further analysis of the power consumption, PDR and throughput is done and shows satisfactory results.

\subsection{AODV Algorithm}

AODV is the routing protocol based on the distance vectorAlgorithm, which integrates the target serial number this protocol mainly, includes routing discovery and routing Maintenance. When the source node communicating with other nodes fails to reach the routing of destination node, it requires the grouping of RREQ. After other nodes receive this RREQ, whether such information exists or not is checked then the information should be abandoned 
International Journal of Trend in Scientific Research and Development (IJTSRD) ISSN: 2456-6470

when necessary. Otherwise, it should record the RREQ in this routing table and broadcast this RREQ continuously until some central node reaches the routing of destination node, or the routing request group in reaches the destination node. In AODV protocol, the node requests for the routing of The destination node through broadcasting RREQ message[5] Gradually. Such a flooding routing method will unfortunately generate substantial RREQ message, resulting in a tremendous signal conflict and the protocol overhead.

\subsection{RSSSI Algorithm}

RSS Generally, the localization algorithms have been proposed, which can be mainly classified into two categories: range-based and range-free. Several rangebased techniques estimate an unknown node distance by three or more beacon nodes. Based on the range information, the location of a node is determined. Some of the range-based localization algorithms include received signal strength indicator (RSSI), angle of arrival (AOA), time of arrival (TOA), and time difference of arrival (TDOA) [4]., the main idea is to estimate the distance of a transmitter to a receiver using the power of received signal, knowledge of the transmitted power the RSSI. The received signal strength from sensor node at node at time is represented By, which is formulated as

$$
P_{r}(d)=P_{T}-\operatorname{PL}\left(d_{0}\right)-10 \eta \log _{10}\left(\frac{d}{d_{0}}\right)+X_{\delta}
$$

Where $P_{r}(d)$ is the received signal, $P_{T}$ is the transmit power, $\mathrm{PL}\left(\mathrm{d}_{\mathrm{o}}\right)$ is the path loss for reference distance of $\mathrm{d}_{0, \eta} \eta$ is the attenuation constant, and $\mathrm{X}_{\delta}=\mathrm{N}\left(0, \delta^{2}\right)$ is the uncertainty factor due to multipath and shadowing.

\section{Simulation results Comparison of Existing system with proposed scheme based on RSSI}

\section{Parameters configuration:}

\begin{tabular}{|l|l|}
\hline CBR Packet Size & 256,512 \\
\hline Transmission Range & $5,20,30,50,100$ \\
\hline Number of Nodes & 50 \\
\hline RSSI & $6.5,7.5$ \\
\hline Simulation Time & 150.0 Seconds \\
\hline
\end{tabular}

\begin{tabular}{|l|l|l|}
\hline TransmissionRange & EHP & AODV \\
\hline 5 & 28.63 & 34.27 \\
\hline 20 & 30.14 & 38.44 \\
\hline 30 & 36.11 & 61.31 \\
\hline 50 & 52.36 & 68.6 \\
\hline 100 & 70.64 & 96.35 \\
\hline
\end{tabular}

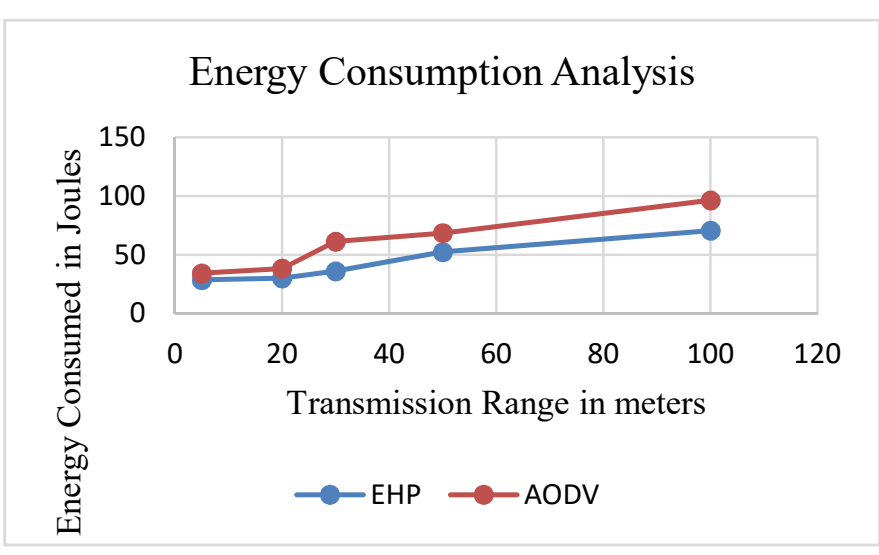

\begin{tabular}{|l|l|l|}
\hline TransmissionRange & EHP & AODV \\
\hline 5 & 24.35 & 37.93 \\
\hline 20 & 32.74 & 54.92 \\
\hline 30 & 36.96 & 59.37 \\
\hline 50 & 68.92 & 75.23 \\
\hline 100 & $\mathbf{8 0 . 3 6}$ & 103.25 \\
\hline
\end{tabular}

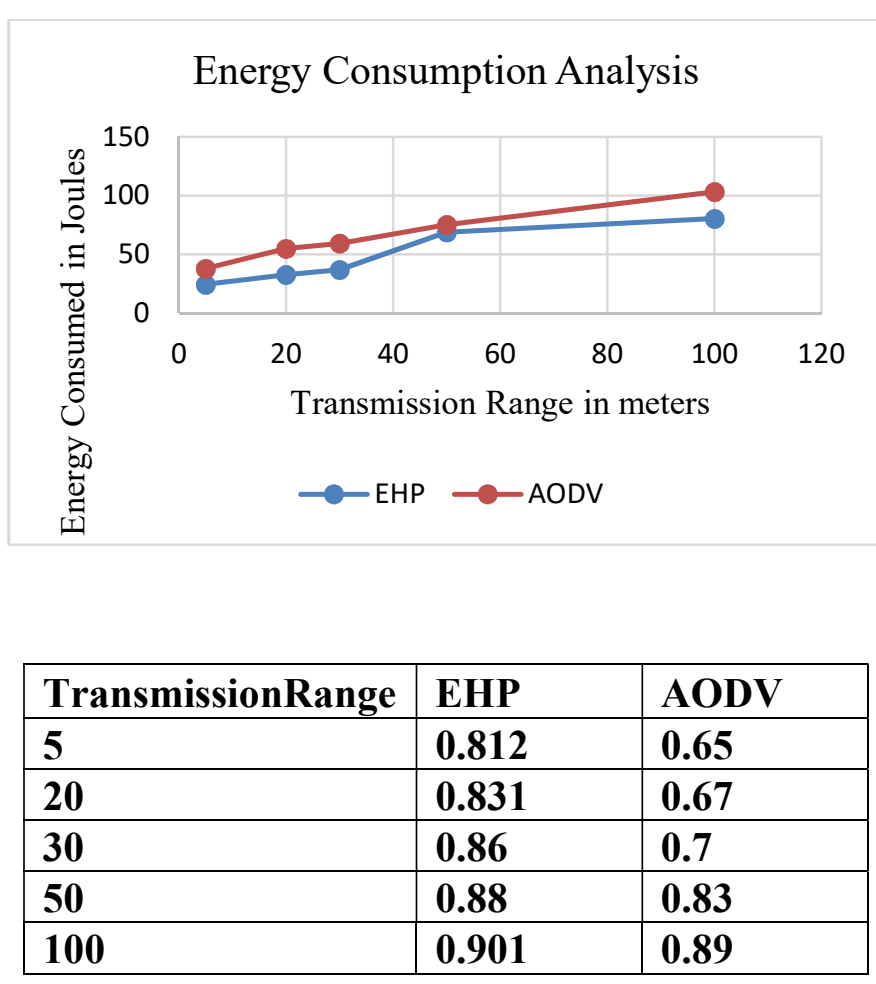


International Journal of Trend in Scientific Research and Development (IJTSRD) ISSN: 2456-6470

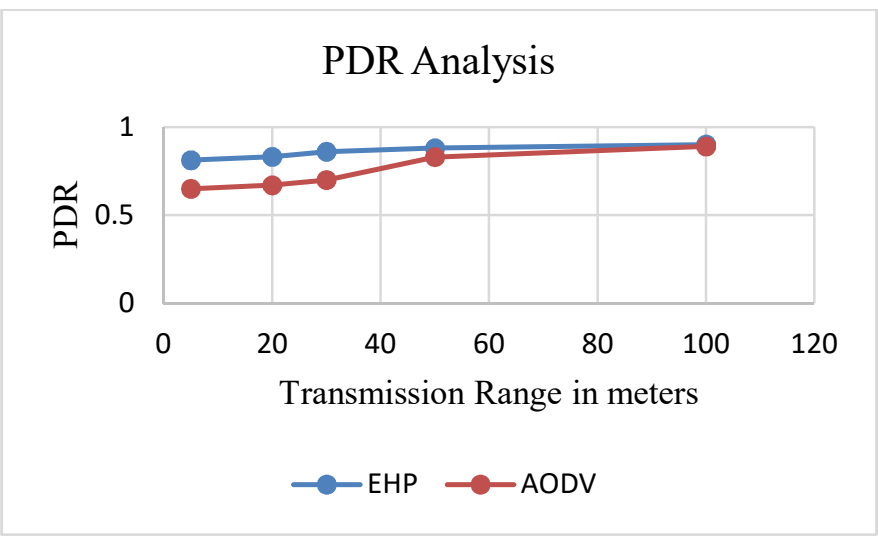

\begin{tabular}{|l|l|l|}
\hline TransmissionRange & EHP & AODV \\
\hline 5 & 104.1 & 111.23 \\
\hline 20 & 151.3 & 150.26 \\
\hline & 270.6 & 210.36 \\
\hline 50 & 297.1 & 234.1 \\
\hline 100 & 326.3 & 280.78 \\
\hline
\end{tabular}

\begin{tabular}{|l|l|l|}
\hline TransmissionRange & EHP & AODV \\
\hline 5 & 0.82 & 0.62 \\
\hline 20 & 0.833 & 0.657 \\
\hline 30 & 0.842 & 0.694 \\
\hline 50 & 0.877 & 0.816 \\
\hline 100 & 0.89 & 0.884 \\
\hline
\end{tabular}
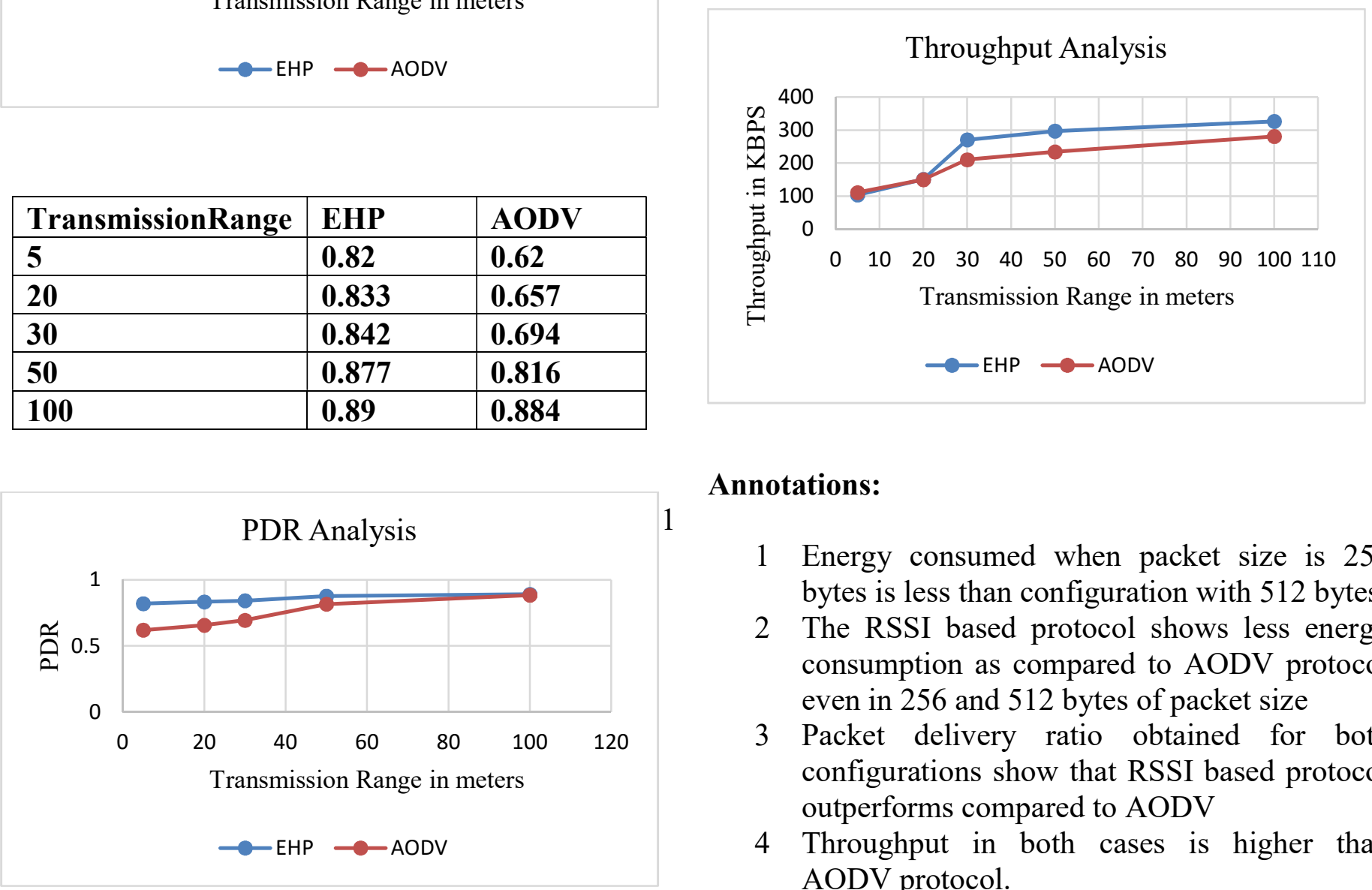

\section{Annotations:}

1 Energy consumed when packet size is 256 bytes is less than configuration with 512 bytes.

2 The RSSI based protocol shows less energy consumption as compared to AODV protocol even in 256 and 512 bytes of packet size

3 Packet delivery ratio obtained for both configurations show that RSSI based protocol outperforms compared to AODV

4 Throughput in both cases is higher than AODV protocol.

5 It can be observed that as transmission range is increased energy consumption in both protocols increases. PDR increases and throughput also increases.

6 Increment in transmission range of each node assures the communication reliability in terms of link establishment process.

7 Increment in transmission range is done by increasing transmission power hence energy consumption is increased with large values.

8 As nodes are coming in contact with each other it assures reliable link between all nodes as far network is formed by the nodes.

9 The effect on interference is also observed when transmission power is increased.

10 When transmission power is increased the RSSI value remains almost higher than the threshold thereby less dropping of packets hence increment in throughput and PDR. 
International Journal of Trend in Scientific Research and Development (IJTSRD) ISSN: 2456-6470

In simulation, RSSI log is created during simulation with respect to time at the time of link establishment procedures. Each log is observed to verify the RSSI based link reliability analysis with respect to transmission power.

\begin{tabular}{|l|l|}
\hline CBR Packet Size & 256,512 \\
\hline Transmission Range & $5,20,30,50,100$ \\
\hline Number of Nodes & 90 \\
\hline RSSI & $6.5,7.5$ \\
\hline Simulation Time & 150.0 Seconds \\
\hline
\end{tabular}

\begin{tabular}{|l|l|l|}
\hline TransmissionRange & EHP & AODV \\
\hline 5 & 36.12 & 45.23 \\
\hline 20 & 38.21 & 51.01 \\
\hline 30 & 46 & 59.81 \\
\hline 50 & 51 & 69.05 \\
\hline 100 & 66.92 & 102.34 \\
\hline
\end{tabular}

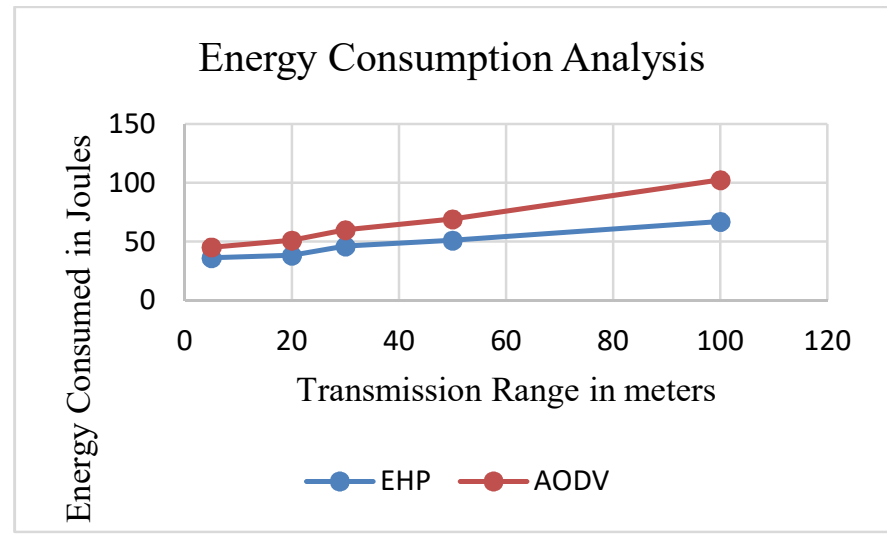

\begin{tabular}{|l|l|l|}
\hline TransmissionRange & EHP & AODV \\
\hline 5 & 31.65 & 51.26 \\
\hline 20 & 41.26 & 58.21 \\
\hline 30 & 50.23 & 65.41 \\
\hline 50 & 66.447 & 80.24 \\
\hline 100 & 79.038 & 96.41 \\
\hline
\end{tabular}

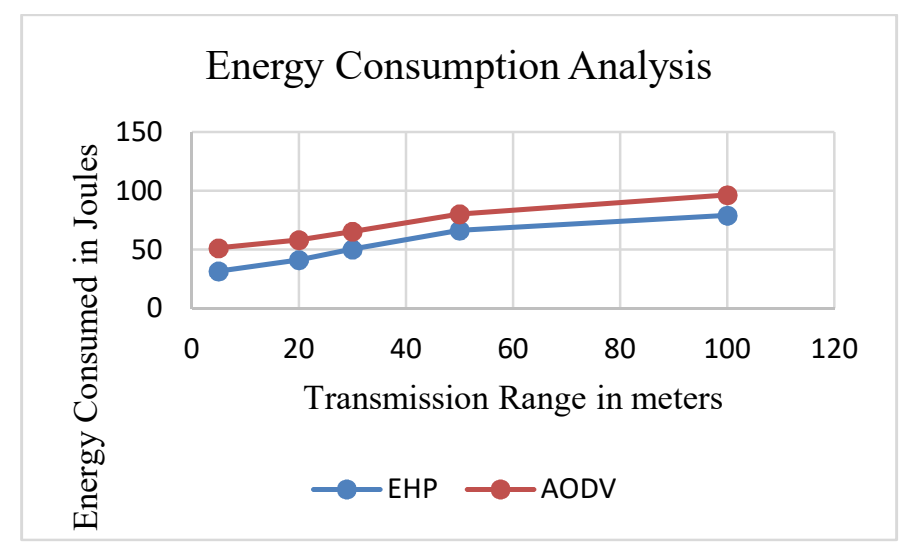

\begin{tabular}{|l|l|l|}
\hline TransmissionRange & EHP & AODV \\
\hline 5 & 0.821 & 0.7 \\
\hline & 0.8311 & 0.72 \\
\hline 30 & 0.8522 & 0.76 \\
\hline 50 & 0.864 & 0.86 \\
\hline 100 & 0.901 & 0.89 \\
\hline
\end{tabular}

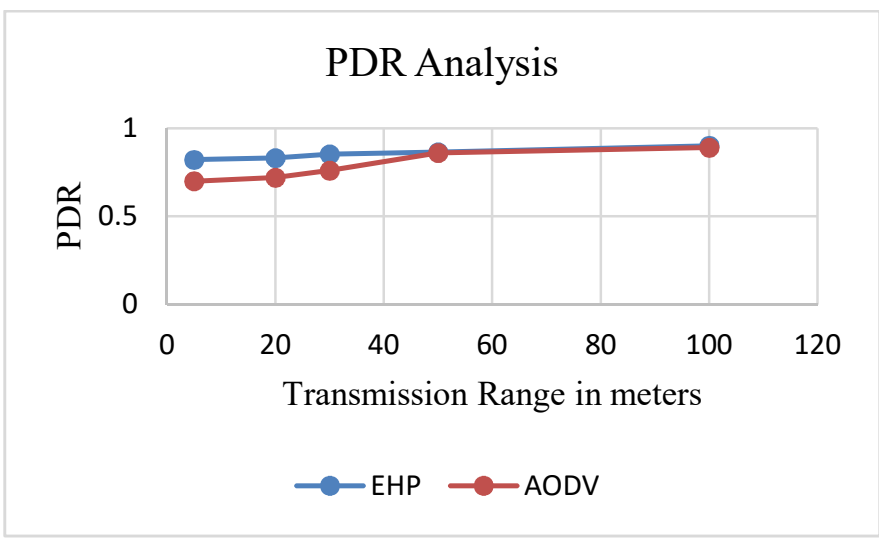

\begin{tabular}{|l|l|l|}
\hline TransmissionRange & EHP & AODV \\
\hline 5 & 0.81 & 0.7 \\
\hline 20 & 0.82 & 0.72 \\
\hline 30 & 0.841 & 0.76 \\
\hline 50 & 0.86 & 0.81 \\
\hline 100 & 0.89 & 0.84 \\
\hline
\end{tabular}

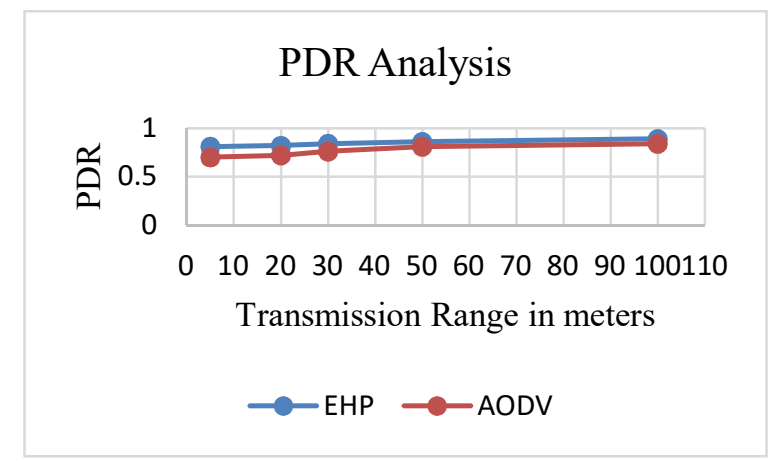

\begin{tabular}{|l|l|l|}
\hline TransmissionRange & EHP & AODV \\
\hline 5 & 290.13 & 290 \\
\hline 20 & 311.21 & 315 \\
\hline 30 & 326.62 & 324 \\
\hline 50 & 411.25 & 356 \\
\hline 100 & 423 & 393 \\
\hline
\end{tabular}




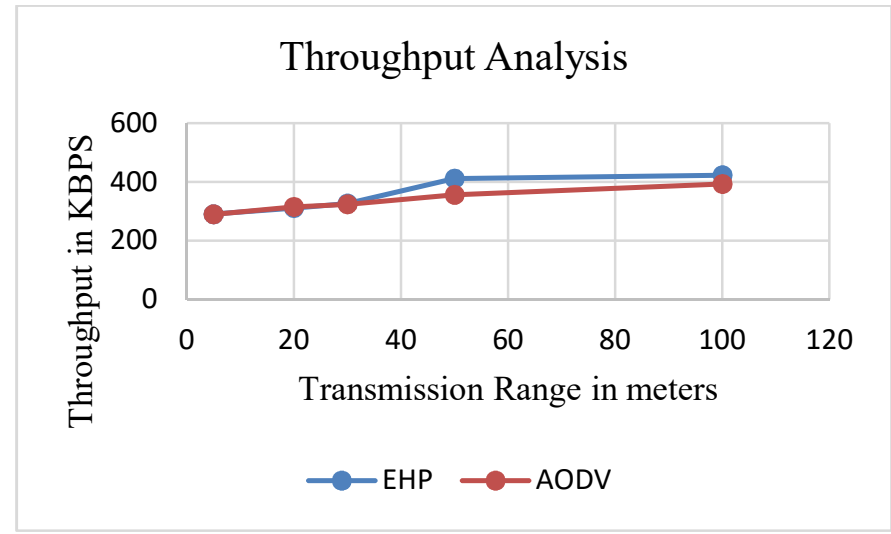

\begin{tabular}{|l|l|l|}
\hline TransmissionRange & EHP & AODV \\
\hline 5 & $\mathbf{3 1 0}$ & $\mathbf{2 7 2 . 3}$ \\
\hline 20 & $\mathbf{3 2 1 . 2 3}$ & $\mathbf{2 8 5}$ \\
\hline 30 & $\mathbf{3 3 5 . 1 4}$ & $\mathbf{3 0 5}$ \\
\hline $\mathbf{5 0}$ & $\mathbf{3 8 0 . 1 5}$ & $\mathbf{3 3 0}$ \\
\hline 100 & 410 & $\mathbf{3 6 5}$ \\
\hline
\end{tabular}

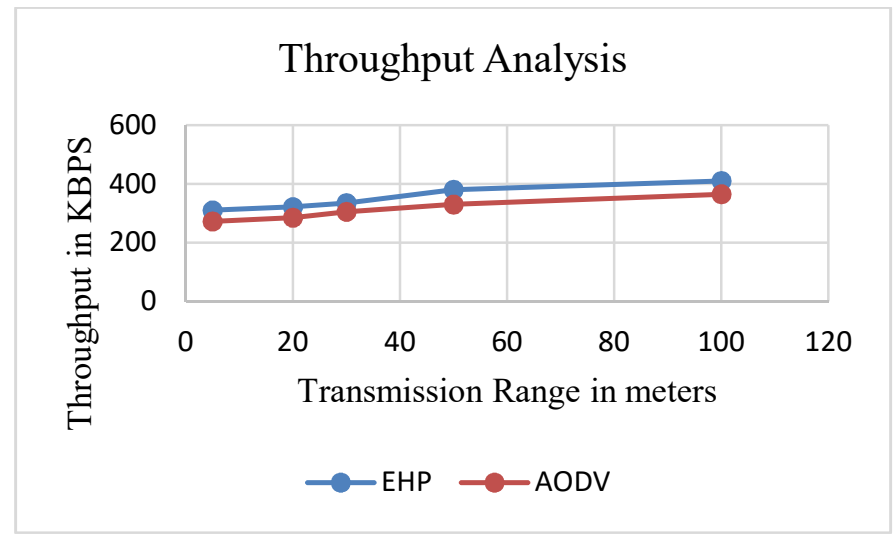

\section{Annotations:}

1 Energy consumed when packet size is 256 bytes is less than configuration with 512 bytes.

2 The RSSI based protocol shows less energy consumption as compared to AODV protocol even in 256 and 512 bytes of packet size

3 Packet delivery ratio obtained for both configurations show that RSSI based protocol outperforms compared to AODV

4 Throughput in both cases is higher than AODV protocol.

5 It can be observed that as transmission range is increased energy consumption in both protocols increases. PDR increases and throughput also increases.

6 Increment in transmission range of each node assures the communication reliability in terms of link establishment process.
7 Increment in transmission range is done by increasing transmission power hence energy consumption is increased with large values.

8 As nodes are coming in contact with each other it assures reliable link between all nodes as far network is formed by the nodes.

9 The effect on interference is also observed when transmission power is increased.

10 When transmission power is increased the RSSI value remains almost higher than the threshold thereby less dropping of packets hence increment in throughput and PDR.

In simulation, RSSI log is created during simulation with respect to time at the time of link establishment procedures. Each log is observed to verify the RSSI based link reliability analysis with respect to transmission power.

\section{Conclusion:}

The proposed method and its comparative analysis with AODV protocol shows that energy consumption, PDR and throughput are optimum and satisfactory in proposed method.

The results show that method outperforms even in case of high density networks. As packet size is changed the impact on parameters is much less compared to AODV protocol.

The proposed method protocol can be used when localization based network establishment is to be done and performance is expected. So far simulation performance is satisfactory.

\section{References}

1) Ahmad El Assaf, Slim Zaidi,SofièneAffes et.al." Low-Cost Localization for Multihop Heterogeneous Wireless Sensor Networks". IEEE transactions on wireless communications, vol. 15, no. 1 , january.

2) D. P. Agrawal and Q.-A. Zeng, Introduction to Wireless and MobileSystems, 3rd ed. Stamford, CT, USA: Cengage Learning, 2010.

3) XihaiZhangJunlongFang,andFanfengMeng“AnEff icientNodeLocalization approach with RSSI for RandomlyDeployedWirelessSensorNetworks"Hin dawi Publishing Corporation Journal of Electrical and Computer Engineering Volume 2016, Article ID 2080854 
4) I. F. Akyildiz and M. C. Vuran, Wireless Sensor Network ,John Wiley \& Sons, London, UK, 2010.

5) "Research on the WSN Node Localization Based on TOA" Qing-huiWang, Ting-ting Lu,Meng-long Liu , and Li-feng Wei Hindawi Publishing Corporation Journal of Applied Mathematics Volume 2013, Article ID 706064,

6) Bo Guan, Xin Li"An RSSI-based Wireless Sensor Network Localization Algorithm with Error Checking and Correction"http://www.i-joe.org 